\title{
Editorial
}

\section{Association of COVID-19 with obesity}

COVID-19 is a disease that cause respiratory illness due to novel corona virus. It was reported to WHO on December 31,2019 for the first time. The outbreak of this disease started from Wuhan city, China. Now COVID-19 pandemic is spreading worldwide mostly in Europe and North America, these regions have high prevalence of obesity.

In the pathogenesis of COVID-19 disease, obesity assumes a significant job. The insusceptible framework, which is official in the pathogenesis of COVID19, assumes a significant job in weight instigated fat tissue aggravation. In the fat tissue the irritation brings about metabolic brokenness conceivably prompting dyslipidemia, type 2 diabetes mellitus, insulin obstruction, hypertension and cardiovascular sickness. Obesity has been expanded the vulnerability to contaminations.

In this pandemic, a large number of obese individual with Covid-19 are reported. Infection rate in obese is greater due to poor immunity, comorbidity and inadequate nutritional needs. Statistical analysis showed that about 41.7\% patients reported in New York city were obese. Whereas $40 \%$ obese have been reported in United State of America with Covid-19. A report from UK indicated that $38 \%$ obese were admitted in ICU with Covid-19. According to Chinese researchers, obese individuals are 3 -times more prone toward the development of Covid-19. So recent analysis indicated that obesity is the major risk factor of Covid-19.

In COVID-19, overweight and obese patients have high danger of metabolic difficulties and eternal infections that stoutness works. More nutrition care is required for such patients. As nutrition is a key factor for keeping up human wellbeing, for example, dense impervious framework and satisfactory admission of supplements and dietary enhancements. Tolerant with COVID-19 create contamination from slight to serious indications bound to the dietary status. Consequently, assessing wholesome status of individuals with contamination turns out to be increasingly significant. Through dietary help, we can bring down the danger of oxidative pressure, infection contamination and expands invulnerability framework among obese people especially. 